\title{
ANÁLISE FENOMENOLÓGICA INTERPRETATIVA (AFI): FUNDAMENTOS BÁSICOS E APLICAÇÕES EM PESQUISA
}

\author{
Interpretative Phenomenological Analysis (IPA): Basic underpinnings and applications in research
}

Análisis Fenomenológico Interpretativo (AFI): Fundamentos básicos y aplicaciones en la investigación

Mário Augusto Tombolato Manoel ANTÔNIO dos Santos

\begin{abstract}
Resumo: No contexto das pesquisas qualitativas, este estudo tem por objetivo apresentar os fundamentos básicos e potenciais aplicações do referencial teórico-metodológico da Análise Fenomenológica Interpretativa (AFI). A AFI é ancorada na articulação de conceitos de três correntes intelectuais: fenomenologia, hermenêutica e idiografia. Este estudo busca apresentar e refletir sobre suas características, potencialidades, limites e, mais especificamente, apresentar e analisar algumas implicações teóricas e práticas da aplicação da AFI em pesquisa. Trata-se de um referencial bem estabelecido internacionalmente, porém pouco difundido na realidade brasileira, onde ainda conta com número restrito de estudos. Com isso, pretende-se contribuir para o preenchimento de uma lacuna da literatura, ampliando o conhecimento dessa abordagem no contexto nacional, de modo a inspirar e incentivar o desenvolvimento de novos estudos.
\end{abstract}

Palavras-chave: Fenomenologia; Hermenêutica; Métodos de Pesquisa - Psicologia; Pesquisa Qualitativa.

\begin{abstract}
In the context of qualitative research, the objective in this study is to address the basic underpinnings and the potential applications of Interpretative Phenomenological Analysis (IPA) as a theoretical-methodological framework. IPA is grounded in concepts and the articulation of three knowledge areas: phenomenology, hermeneutics and idiography. This text deals with the introduction and characteristics, potentialities, limitations of, and - more specifically - introduce and discusses theoretical and practical IPA applications in research. Although it is well established internationally, a limited number of studies in Brazil are grounded in IPA. Therefore, this paper is expected to contribute to the dissemination of the approach in the Brazilian context by filling the existing gap and, as a result, inspire and encourage the development of new studies.
\end{abstract}

Keywords: Phenomenology; Hermeneutics; Research Methods - Psychology; Qualitative Research.

Resumen: En el contexto de las investigaciones cualitativas, este estudio tiene por objetivo presentar los fundamentos básicos y posibles aplicaciones del referencial teórico-metodológico del Análisis Fenomenológico Interpretativo (AFI). La AFI es anclada en la articulación de conceptos de tres áreas del conocimiento: fenomenología, hermenéutica e idiografía. Este estudio busca presentar y reflexionar sobre sus características, potencialidades, límites y, más específicamente, presentar y analizar algunas implicaciones teóricas y prácticas de la aplicación de la AFI en investigación. Se trata de un referencial bien establecido internacionalmente, pero poco difundido en la realidad brasileña, donde cuenta con número restringido de investigaciones. Con ello, se pretende contribuir al llenado de una laguna de la literatura, ampliando el conocimiento de ese abordaje en el contexto nacional, para inspirar e incentivar el desarrollo de nuevos estudios.

Palabras-clave: Fenomenología; Hermenéutica; Métodos de Investigación - Psicología; Búsqueda cualitativa.

\section{Introdução}

As pesquisas científicas podem ser classificadas de variadas formas, a depender de sua natureza, área, abordagem e também do paradigma, propósito e procedimentos metodológicos utilizados para alcançar os objetivos propostos (Pernecky, 2016; Savin-Baden \& Major, 2013). O método descreve o caminho trilhado pelo(a) pesquisador(a) em sua investigação. Pode ser definido como um conjunto de "procedimentos que consideramos adequados para responder à nossa questão; não é um a priori da pesquisa, ele faz parte dela” (Furlan, 2008, p. 25).

Em uma aproximação inicial, as atividades do campo científico podem ser classificadas em dois grandes eixos: pesquisas teóricas e pesquisas empíricas. Basicamente, as pesquisas teóricas têm por objetivo a construção, revisão e reconstrução de teorias, conceitos e ideologias. Já as pesquisas empíricas se caracterizam pela coleta de dados a partir de fontes diretas - pessoas que conhecem, vivenciaram ou têm conhecimento sobre o tema e/ou situação investigada (Fonseca, 2002).

As pesquisas empíricas podem ser classificadas em quantitativas ou qualitativas. De modo geral, a abordagem quantitativa usa os princípios da confiabilidade da medida para descrever e avaliar o rigor 
científico do estudo, enquanto que, na abordagem qualitativa, o rigor está relacionado ao intuito de buscar comprender o conhecimento a partir da concepção particular da realidade dos(as) participantes $(\mathrm{Wu}$, Thompson, Aroian, McQuaid, \& Deatrick, 2016).

A investigação qualitativa pode ser entendida como uma abordagem metodológica ampla, que engloba diversos métodos de pesquisa. Os objetivos pretendidos variam muito nas diferentes áreas do conhecimento. A abordagem qualitativa é utilizada em diferentes campos disciplinares, porém é mais disseminada nas ciências humanas e sociais, como a psicologia, educação, antropologia, ciência política e serviço social. Isso considera o fato de que os métodos qualitativos compõem uma paisagem heterogênea e extremamente diversificada (Creswell, 2008; Pernecky, 2016), devendo ser buscado aquele que tem maior pertinência com o fenômeno de investigação. Desse modo, há múltiplas e distintas metodologias qualitativas, sendo cada uma ancorada em diferentes pressupostos ontológicos e epistemológicos, tais como: abordagem narrativa, sócio histórica, fenomenologia, etnografia, estudo de caso, teoria fundamentada nos dados, teoria crítica, construtivismo, pós-positivismo, entre outros enfoques que se afinam estreitamente às exigências metodológicas das ciências humanas e sociais.

No campo da psicologia, quando o(a) investigador(a) intenciona obter uma compreensão em profundidade de determinado comportamento e escrutinar as variáveis que o regulam, ele(a) pode ou não lançar mão de métodos qualitativos ${ }^{1}$. Esses métodos possibilitam examinar como e por quê foi tomada determinada decisão, não apenas o quê, onde, quando ou quem emitiu aquela conduta. Nessa perspectiva, os métodos qualitativos produzem informações minuciosas sobre os casos particulares investigados, sem almejar generalizações (Creswell, 2008; Denzin \& Lincoln, 2005).

De fato, na pesquisa qualitativa quaisquer conclusões mais gerais devem ser vistas com cautela e consideradas como proposições ou pressupostos informados, não como hipóteses que são formuladas, por exemplo, pelos princípios da causalidade e testagem, característicos dos métodos quantitativos. A relevância da pesquisa qualitativa está justamente na sua capacidade de examinar a fundo determinado campo de investigação. Por outro lado, os métodos quantitativos podem ser utilizados para buscar apoio empírico para tais pressupostos de pesquisa (Lindlof \& Taylor, 2002). Desse modo, resguardadas

1 A relação estreita entre o(a) pesquisador(a) e seu campo de estudo é uma característica intrínseca à investigação social. Desse modo, parte-se do pressuposto de que a visão de mundo de quem investiga e a do sujeito da pesquisa estão implicadas não apenas no produto final da investigação, mas desde o princípio da concepção do objeto até os resultados obtidos e sua potencial aplicação. Logo, é essencial que o(a) pesquisador(a) conheça a complexidade dos tipos de estudos, os diversos delineamentos de pesquisa, para então definir quais os instrumentos e procedimentos que melhor se adequarão aos propósitos de sua investigação (Kauark et al., 2010). suas especificidades, não há antagonismo entre os métodos quali e quantativos, mas complementaridade (Minayo \& Sanches, 1993).

$\mathrm{O}(\mathrm{a})$ investigador(a) qualitativo(a) frequentemente não tem constituída a compreensão prévia acerca do fenômeno pesquisado (Denzin \& Lincoln, 2005). Pode partir de um pré-reflexivo, como na investigação de inspiração fenomenológica, ou pode lançar mão de uma abordagem teoricamente fundamentada, como a grounded theory. Pode ainda aplicar os princípios balizadores do estudo de caso, com a finalidade de examinar em profundidade amostras de conveniência (ou intencionais) para explorar um determinado fenômeno (Stake, 1995).

De acordo com Bogdan e Taylor (1990), o(a) pesquisador(a) pode iniciar seu estudo com proposições gerais acerca da realidade a ser investigada e proceder de forma científica e empírica ao longo do processo de investigação. A compreensão abrangente de um fenômeno, evento ou situação deriva da exploração da totalidade da situação. Por isso, o recorte a ser feito no estudo necessariamente tem de ser bem delimitado, ainda que esse procedimento possa permitir o acesso a uma quantidade considerável de dados que precisam ser ordenados.

$\mathrm{Na}$ atualidade, a pesquisa qualitativa utiliza um número amplo de paradigmas, que refletem as preocupações conceituais e teóricas dos(as) investigadores(as) contemporâneos (Pernecky, 2016). Questões identitárias, por exemplo, que emergiram com força a partir da década de 1980 e que podem incluir problemáticas relacionadas à etnia/"raça"/cor da pele, classe, gênero, diversidade cultural e comunidades discursivas, levaram a pesquisa no campo das ciências sociais a se tornar cada vez mais reflexivas e aprofundadas. Com isso, os pressupostos teóricos, ontológicos e epistemológicos são, cada vez mais (e melhor) contextualizados pelos(as) pesquisadores(as). O que se valoriza é a busca de legitimidade junto à comunidade científica, principalmente frente a outros paradigmas mais consolidados historicamente, como os derivados do positivismo e de outras matrizes teórico-epistemológicas. Entende-se que a garantia de rigor na análise dos dados, a reflexividade em todas as etapas da pesquisa e a descrição densa e minuciosa dos procedimentos utilizados são, em última instância, os aspectos que garantem a legitimidade do método empregado (Wu, et al., 2016).

As metodologias qualitativas podem ser compreendidas como uma família de abordagens que compartilham de certas características que são comuns e que as distinguem da pesquisa quantitativa (Wu, et al., 2016). Nessa perspectiva, pode-se afirmar que "a pesquisa qualitativa se ocupa com o universo dos significados, dos motivos, das aspirações, das crenças, dos valores e das atitudes" (Minayo, 2010, p. 21). Com isso, possibilita uma visão compreensivo-interpretativa do fenômeno estudado por meio do vértice do vínculo direto e intersubjetivo 
estabelecido entre o(a) pesquisador(a) e os(as) participantes (Lüdke \& André, 1986).

Na paisagem heterogênea das abordagens qualitativas contemporâneas, o termo "pesquisa interpretativa" tem sido utilizado para nomear os estudos de delineamento qualitativo e as pesquisas indutivas (Lowenberg, 1993). A visão compreensivo-interpretativa, conforme pontuada por Minayo (2010), decorre do reconhecimento básico de que no ato de pesquisar estão envolvidos os processos interpretativos e cognitivos inerentes à vida social. Esses processos são fortemente enfatizados nessas abordagens.

Ademais, a investigação qualitativa tem determinadas características que são cruciais ao cenário a ser estudado, podendo-se destacar: a preocupação com o contexto no qual os indivíduos estão inseridos; a valorização da descrição densa dos dados obtidos, que devem ser analisados em toda a sua profundidade e riqueza; o interesse do(a) pesquisador(a) pelo processo em vez de focar simplesmente nos resultados; e a relevância do significado que cada participante atribui às experiências vividas. Nessa vertente, Guba e Lincoln (2005) identificam cinco paradigmas principais da pesquisa qualitativa contemporânea: positivismo, pós-positivismo, teorias críticas, construtivismo e paradigmas participativos-cooperativos. Essas vertentes refletem diferenças axiomáticas, objetivos propostos, controle de processos e desfechos de pesquisa, relação com fundamentos de verdade e conhecimento, representação textual, validade e voz do(a) pesquisador(a) e dos(as) participantes. Desse modo, a pesquisa qualitativa visa a ampliar o conhecimento sobre o fenômeno em questão por meio de análise realizada a partir do levantamento de informações originárias dos relatos dos(as) próprios(as) participantes (Lüdke \& André, 1986).

No panorama qualitativo de pesquisa, nota-se a existência de variados tipos de pesquisa fenomenológica. Amatuzzi (1996) evidencia seis deles: (1) pesquisa fenomenológica como filosofia, que visa a esclarecer o conhecimento a partir da descrição daquilo que se mostra, abstendo-se de juízos a priori e sem concepções explicativas; (2) fenomenologia eidética, que elucida vivências por meio da reflexão e redução fenomenológica, (3) fenomenologia hermenêutica, na qual a compreensão do vivido se dá pela interpretação do(a) pesquisador(a), (4) psicologia fenomenológica "empírica", que aplica a filosofia fenomenológica aos estudos em psicologia com dados empíricos, (5) pesquisa psicológica "experimental", que combina a fenomenologia empírica com a intervenção do método experimental, e (6) pesquisa colaborativa, na qual a pesquisa fenomenológica é aplicada aos fenômenos do processo grupal. No presente estudo focalizamos, apresentamos e analisamos reflexivamente a abordagem teórico-metodológica da Análise Fenomenológica Interpretativa - AFI, uma proposta recente de sistematização da investigação científica em pesquisa qualitativa, que se insere também no paradigma fenomenológico.

Este estudo tem por objetivo apresentar, no contexto das pesquisas qualitativas, os fundamentos básicos e algumas aplicações do referencial teórico-metodológico da Análise Fenomenológica Interpretativa - AFI, e refletir sobre suas características, potencialidades e limites. Mais especificamente, pretende-se analisar algumas implicações teóricas e práticas da aplicação da AFI à pesquisa.

\section{Método}

Trata-se de um estudo de caráter teórico-metodológico, que aborda os pilares de uma proposta recente de compreensão fenomenológica, sistematizada a partir da aproximação com aportes da hermenêutica e da idiografia. Estudos teórico-metodológicos se justificam por iluminar determinado campo de investigação que apresenta potencial de inovação e reflexão metodológica, considerando a dinâmica criativa das teorias e metodologias de pesquisa. Estas teorias/metodologias caracterizam um universo que está sempre em expansão, na medida em que novos desafios se apresentam para o campo da pesquisa em ciências humanas e sociais.

\section{Análise Fenomenológica Interpretativa - AFI: contexto e marco teórico-conceitual}

O conteúdo apresentado nesta seção refere-se, principalmente, ao contexto teórico da abordagem. As reflexões foram inspiradas no principal material que a fundamenta, o livro Interpretative Phenomenological Analysis: theory, method and research, de Jonathan A. Smith, Paul Flowers e Michael Larkin. Essa obra seminal foi publicada no ano de $2009 \mathrm{em}$ língua inglesa e ainda não se encontra traduzida para a língua portuguesa. Trata-se de uma referência primordial, que oferece um painel amplo e detalhado que serve como guia sobre os aspectos teóricos, metodológicos e aplicados da AFI (acrônimo em português para Interpretative Phenomenological Analysis - IPA), que serão explorados neste estudo (Smith, Flowers \& Larkin, 2009). Os autores da AFI são psicólogos e professores de universidades britânicas, respectivamente Birkbeck University of London, Glasgow Caledonian University e University of Birmingham.

De acordo com Smith et al. (2009), a AFI é uma abordagem desenvolvida na segunda metade da década de 1990 para subsidiar a pesquisa qualitativa, experiencial e psicológica. É originária do campo da psicologia, mas também tem sido adotada progressivamente nas ciências humanas, sociais e de saúde. Esse referencial teórico-metodológico tem o propósito de investigar como as pessoas dão sentido às suas experiências de vida mais significativas.

A AFI despontou em 1996, com a publicação de um artigo de Smith (1996) no periódico Psychology 
and Health, no qual o autor propôs uma abordagem em psicologia que pudesse capturar a experiência qualitativa, mantendo um diálogo com a psicologia tradicional. Como já mencionado, a AFI teve seu ponto de partida na psicologia e a maioria das pesquisas iniciais foi em psicologia da saúde. Desde então, ela tem expandido seu campo de aplicação e adquirido força na psicologia clínica e social, assim como no aconselhamento psicológico. Ademais, a AFI também está começando a ser utilizada em disciplinas afins nas ciências humanas, da saúde e sociais (Smith et al., 2009).

A AFI é baseada em conceitos e articulações de três correntes intelectuais: fenomenologia, hermenêutica e idiografia, que serão apresentadas a seguir, de maneira básica e esquemática, de acordo com os autores, com o intuito de fornecer um panorama geral das bases teórico-filosóficas. Vale ressaltar que não é a única abordagem de pesquisa que trabalha utilizando uma combinação dessas áreas do conhecimento e, embora haja características centrais da AFI, há variadas formas delas se apresentarem nos estudos. Nesse sentido, por exemplo, Packer e Addison (1989) apresentaram diversas pesquisas em psicologia relacionadas às perspectivas hermenêutica e interpretativa.

Além disso, é interessante situar a AFI em relação à abordagem da Psicologia Fenomenológica proposta por Amedeo Giorgi (Giorgi \& Sousa, 2010), considerada a mais tradicional no campo da Psicologia. Ambas são similares pois tentaram operacionalizar a fenomenologia para a psicologia e, nesse empreendimento intelectual, realizaram transformações que foram necessárias quando se transpõe as contribuições oriundas da filosofia para a psicologia. A diferença mais importante entre a Psicologia Fenomenológica e a AFI é que Giorgi propôs uma articulação mais próxima ao método fenomenológico de Husserl, enquanto que a AFI utilizou um corpus mais abrangente de autores do campo da fenomenologia, sem a intenção de operacionalizar qualquer uma das correntes que tomou em consideração. Outra diferença fundamental é que a AFI é, majoritariamente, interpretativa, enquanto que a Psicologia Fenomenológica de Giorgi é descritiva (Giorgi \& Sousa, 2010; Smith et al., 2009).

\section{Fenomenologia}

Como escola filosófica, a fenomenologia despontou no final do século XIX, na Alemanha, com a obra Investigações lógicas (Logische Untersuchungen) do filósofo e matemático Edmund Husserl (Husserl, 2012; Moreira, 2002). A fenomenologia se define como ciência dos fenômenos, isto é, um campo científico que almeja investigar aquilo que aparece (ou se revela) a uma dada consciência e como aparece, bem como as estruturas subjacentes a esse aparecer. Desse modo, fenômeno é definido como aquilo que é revelado à consciência. A fenomenologia questio- na a ênfase atribuída pela ciência positivista do século XIX à exatidão, objetividade e neutralidade do conhecimento, que somente poderia ser alcançado a partir de métodos experimentais fundamentados nas ciências naturais, legitimados como único modo para se chegar a uma verdade (Forghieri, 2004; Heidegger, 2012). Husserl partilha desses ideais positivistas, porém, no que se refere à neutralidade, para garanti-la, ele avança em relação ao conhecimento propondo o conceito de epochê.

Três dos maiores filósofos do século XXI, Heidegger, Sartre e Merleau-Ponty, inspiraram-se, cada qual à sua maneira, nos pressupostos fenomenológicos de Husserl para suas investigações (Feijoo \& Mattar, 2014). Cada um deles constituiu uma perspectiva particular e distinta, que para Feijoo e Mattar é consistente com a fenomenologia central ${ }^{2}$. Smith et al. (2009), a partir de algumas ideias seminais de Husserl, Heidegger, Sartre e Merleau-Ponty, apontam os principais desenvolvimentos na fenomenologia, que constituem os pilares mais relevantes para os(as) pesquisadores(as) da AFI.

A princípio, o trabalho de Husserl estabelece a relevância no foco da experiência e a sua percepção (assim como seu conceito de Lebenswelt). Com isso, consciência e sujeito são vistos como indissolúveis e indissociáveis (Husserl, 2012). Posteriormente, Heidegger, Merleau-Ponty e Sartre, cada um ao seu modo, contribuem para uma visão de pessoa como envolvida e imersa em um mundo de objetos e relacionamentos, linguagem e cultura, projetos e preocupações. Esses autores nos movem para uma posição distante dos compromissos descritivos e dos interesses transcendentais de Husserl, caminhando em direção a uma posição mais interpretativa e relacionada ao mundo, com foco no entendimento da direção do nosso mundo vivido - perspectiva que é pessoal para cada um de nós, mas que também é uma propriedade de nossos relacionamentos com o mundo e com os outros, em vez de ser para nós como criaturas isoladas. Assim, no vislumbre da obra monumental desses autores, podemos identificar que a compreensão complexa da "experiência" invoca um processo vivido, um desdobramento de perspectivas e sentidos que são únicos para o corpo, e o relacionamento situado da pessoa no mundo (Smith et al., 2009).

De acordo com os referidos autores, tal fato nos autoriza a situar a fenomenologia como uma postura singular, mas também com um alcance pluralista: há muitas e diferentes ênfases e interesses entre os(as) fenomenólogos(as), porém todos(as) eles(as) tendem a compartilhar a trajetória do pensamento sobre como é a experiência do ser humano) ${ }^{3}$ em todos os

2 Heidegger não assumiu o método husserliano; não há epochê, nem variação eidética, assim como não há intuição de essências em sua obra. Os casos de Sartre e Merlau-Ponty são mais complexos, mas pairam dúvidas sobre se eles de fato seguiram o método husserliano. 3 Aqui cabe uma ponderação frente a essa assertiva de Smith et al. (2009). Husserl não está interessado somente na experiência de ser humano, mas sim nas estruturas essenciais de toda experiência possível. 
seus variados aspectos, especialmente em termos das coisas que nos importam e constituem o nosso mundo vivido.

A AFI é fenomenológica no que se refere à investigação da experiência em seus próprios termos. Experiência também pode ser compreendida como sinônimo de vivência. Nesse sentido, considera-se vivência (Erlebnis, originalmente em alemão) de acordo com esses aspectos: “a percepção que o ser humano tem de suas próprias experiências, atribuindo-lhes significados [...,] acompanhadas de algum sentimento de agrado ou desagrado" (Forghieri, 1993, p. 19). Logo, "o que é vivenciado deve ter uma intensidade de tal modo significativa, cujo resultado confere uma importância que transforma por completo o contexto geral da existência” (Viesenteiner, 2013, p. 142). Embora vivência/experiência seja um conceito complexo, os(as) pesquisadores(as) em AFI estão interessados(as) justamente naquilo que acontece quando o transcorrer das experiências de vida assume um significado particular para as pessoas (Smith et al., 2009).

Assim, os autores chamam a atenção para a existência de uma hierarquia de experiências, na qual o nível mais básico representaria a ausência de consciência própria acerca de nossa absorção no fluxo cotidiano da experiência. $\mathrm{Na}$ medida em que nos tornamos conscientes do que está acontecendo, nós temos o início do que pode ser descrito, pelos autores, como "uma experiência” em oposição simplesmente à “experiência”. Quando as pessoas estão envolvidas com "uma experiência” de algo maior em suas vidas, independentemente de ela ser valorada como positiva ou negativa, elas começam a refletir sobre o significado do que está acontecendo, e a pesquisa na AFI tem por objetivo envolvê-las nessas reflexões (Larkin, Watts, \& Clifton, 2006).

$\mathrm{Na}$ pesquisa inspirada na AFI, as tentativas de compreender as relações das pessoas com o mundo são necessariamente interpretativas, e vão focalizar as suas tentativas de atribuir significado para as atividades e coisas que acontecem com elas, na medida em que acontecem. Por esse motivo, é necessário discutir o segundo campo de conhecimento que informa a AFI, a hermenêutica, que focaliza a questão da interpretação propriamente dita.

\section{Hermenêutica}

É uma parte importante da história intelectual, que se dedica ao estudo da teoria da interpretação, ou seja, desde a arte da interpretação até a prática, treino e processo interpretativo (Gadamer, 1999; Ricoeur, 1970). Por isso a hermenêutica contribui para moldar visões teóricas na AFI, que é uma abordagem fenomenológica interpretativa e, portanto, a apropriação de Heidegger em relação à fenomenologia como um empreendimento hermenêutico é consubstancial. Nesse sentido, a AFI se insere na investigação de um fenômeno que se apresenta, e o(a) pesquisador(a) está implicado na facilitação e compreensão dessa manifestação (Smith et al., 2009).

Nas pesquisas fundamentadas na AFI, a tentativa do(a) participante de dar sentido ao que está acontecendo consigo leva o(a) pesquisador(a) a uma perspectiva interpretativa, portanto, orientada pela hermenêutica. Os seres humanos têm uma vida mental e emocional interna. A linguagem, enquanto manifestação empírica, é a uma rica expressão da vida interna de uma pessoa (Schmidt, 2012). Assim, a AFI compartilha a visão de que os seres humanos são criaturas "produtoras de sentido", portanto, os relatos que os(as) participantes apresentam vão refletir as suas tentativas de dar sentido às suas experiências. Desse modo, a AFI também reconhece que o acesso à experiência é sempre dependente daquilo que os(as) participantes relatam sobre suas experiências, e que o(a) pesquisador(a) caminha no sentido de interpretar o relato do(a) participante para se aproximar da compreensão de sua experiência (Smith et al., 2009). "A hermenêutica enquanto compreensão interpretativa das expressões linguísticas é o modelo para o processo geral de compreensão nas ciências humanas" (Schmidt, 2012, p. 21).

Segundo os autores, podemos afirmar que o(a) pesquisador AFI envolve-se em uma hermenêutica dupla, porque está tentando dar sentido à tentativa do participante dar sentido ao que está acontecendo com ele. Ao se posicionar dessa maneira, o investigador(a) está, portanto, empregando as mesmas capacidades mentais e habilidades pessoais que o(a) participante, com quem ele compartilha uma propriedade fundamental, a de ser humano. Ao mesmo tempo, o(a) pesquisador(a) emprega suas habilidades de modo mais autoconsciente e sistemático. Desse modo, o processo de dar sentido - que é parte da tarefa do(a) pesquisador(a) - é de segunda ordem; na medida em que o(a) investigador(a) tem acesso apenas à experiência do(a) participante por meio de seu próprio relato. Essa delimitação circunscreve os limites e possiblidades do trabalho interpretativo, o que leva à necessidade de incorporar à AFI as questões da Idiografia.

\section{Idiografia ${ }^{4}$}

A AFI compromete-se com a investigação detalhada de um caso em particular. Almeja conhecer em detalhe como é a experiência e o sentido que uma pessoa, em particular, atribui ao que está acontecendo com ela. Por isso, afirma-se que a AFI é idiográfica (Smith et al., 2009). Ela adota procedimentos analíticos para, a partir dos casos singulares,

4 Campo de investigação científica que trata de estudos dedicados à compreensão das particularidades individuais e únicas dos objetos de estudo. A abordagem idiográfica está relacionada com casos singulares, tal qual em um estudo de caso. $\mathrm{O}$ conceito foi originalmente proposto pelo filosofo neokantiano Wilhelm Windelband, que introduziu os termos nomotético e idiográfico, os quais, posteriormente, foram utilizados em psicologia e em outras áreas do conhecimento, embora não necessariamente mantendo, nesse processo de apropriação, seu significado original. 
seguir em direção a afirmações mais genéricas, mas que ainda permitem que se recuperem elementos específicos dos participantes da pesquisa. A idiografia contrasta com a maioria do conhecimento em psicologia, que é nomotético, ou seja, os(as) pesquisadores(as) estão mais interessados(as) em pesquisas em nível grupal ou populacional, como via de acesso ao estabelecimento de leis gerais do comportamento humano (Smith et al., 2009). A idiografia tem um papel importante a desempenhar na psicologia e no desenvolvimento de modelos de estudos fenomenológicos - para a síntese de múltiplas análises de pequenos estudos e casos singulares.

A AFI está comprometida com o particular em dois níveis: o particular em detalhes com análise em profundidade, e o conhecer como determinado fenômeno foi compreendido da perspectiva particular de uma pessoa, em um contexto específico. Com isso, os estudos com a AFI normalmente têm um número condensado de participantes. O objetivo não é sistematizar o modo como um número extenso de indivíduos opera para atribuir sentido às suas vivências, mas revelar algo sobre a experiência de cada um. Como parte disso, o estudo pode explorar detalhadamente as convergências e divergências entre os casos, ou seja, as semelhanças e diferenças observadas entre os casos investigados. É possível ir em direção à formulação de alegações mais genéricas, porém isso só deve acontecer após realizada a análise de cada caso (Smith et al., 2009).

De modo geral, a AFI está compromissada com a avaliação detalhada da experiência humana vivida (Larkin et al., 2006). Ela tem por finalidade conduzir a avaliação de forma que permita que a experiência seja expressa em seus próprios termos, em vez de apelar para a construção de sistemas de categorizações pré-definidas. Isso é o que faz a AFI fenomenológica e a conecta a um núcleo de ideias unificadoras que atravessaram as obras de filósofos e fenomenólogos, conforme discutido anteriormente.

A AFI está em consonância com o pensamento de Heidegger ao admitir que a pesquisa fenomenológica é, desde o início, um processo interpretativo. A AFI também busca um compromisso idiográfico situando os(as) participantes nos contextos particulares em que se encontram inseridos(as) e lançados(as), explorando suas perspectivas pessoais e iniciando com uma avaliação detalhada de cada caso antes de ir em direção a alegações gerais. Assim, a AFI se relaciona a cada uma das três correntes intelectuais descritas previamente (Smith et al., 2009).

É válido ressaltar que a sistematização dessa abordagem foi determinada por concepções teóricas reconhecidas como importantes e reflete uma tentativa de operacionalizar um modo de trabalhar pragmaticamente com essas ideias, trazendo-as para o contexto da análise da experiência vivida em contextos como o de saúde e educação. Não é a única abordagem de pesquisa que tenta articular ideias extraídas dos campos da fenomenologia e da hermenêutica, e também não deve ser vista como uma perspectiva que tenta abstrair do contexto original e fixar a experiência em um esquema interpretativo. Pelo contrário, a preocupação recai justamente em um esforço de reconstrução do contexto original, no qual a experiência se inscreve de forma concreta e encarnada.

Considerar o arcabouço teórico subjacente à AFI é tão importante quanto as questões relacionadas ao uso de procedimentos. Pesquisadores(as) que se familiarizam com essa abordagem serão capazes de desenhar as suas compreensões filosóficas que os(as) ajudam a resolver problemas imprevistos, e conforme cresçam sua confiança e experiência, poderão desenvolver criativamente seu trabalho com apoio da AFI, mas de forma que vá além dos procedimentos já descritos. É relevante frisar que essa abordagem não tenta operacionalizar uma ideia filosófica específica, já que recorre a uma gama robusta de postulados extraídos de diversas correntes intelectuais. Segundo os autores, os(as) pesquisadores(as) precisam conhecer minimamente a história da fenomenologia e da hermenêutica para serem capazes de situar a AFI na trajetória do movimento intelectual. Inclusive para delimitarem a diferença entre a AFI e outros métodos fenomenológicos e hermenêuticos aplicados à Psicologia, considerando que a AFI não é o único método a se apoiar nessas tradições.

\section{Aspectos metodológicos e aplicabilida- de da AFI}

Ao eleger a AFI como o referencial teórico-metodológico que poderá nortear uma pesquisa, o(a) pesquisador(a) tem em mente que pretende investigar, descrever, contextualizar e interpretar os significados que os(as) participantes atribuem às suas vivências.

Estudos com a AFI são conduzidos a partir de um pequeno número de participantes e o objetivo do(a) pesquisador(a) é encontrar um grupo razoavelmente homogêneo em determinada característica, de forma que seja possível avaliar a convergência e divergência entre certos aspectos na experiência vivida pelos(as) participantes da pesquisa. Portanto, as conclusões são limitadas ao grupo estudado, mas uma certa extensão pode ser considerada por meio de uma generalização teórica, na qual o leitor pode ser capaz de avaliar a evidência em relação ao seu conhecimento profissional e experiencial. Nesse sentido, pesquisas fundamentadas por esse referencial teórico-metodológico não têm o propósito de definição de amostragens representativas de participantes (Smith et al., 2009). Segundo Denzin e Lincoln (2005), na investigação qualitativa o princípio da generalização estatística dos achados para a população, a partir da investigação do comportamento de uma amostra representativa de sujeitos, é substituído nas pesquisas qualitativas pelo pressuposto da 


\section{generalização teórica ${ }^{5}$ \\ Da entrevista semiestruturada à entre- vista aberta}

Segundo o referencial da AFI, a coleta de dados acontece principalmente na forma de entrevistas semiestruturadas, em que um roteiro de entrevista é flexível e o(a) participante desenvolve uma interação significativa com o tema abordado pelo(a) entrevistador(a). Quando o(a) pesquisador(a) se torna mais experiente, pode escolher por conduzi-las sem utilização de um roteiro estruturado ou semiestruturado, privilegiando a entrevista aberta. Assim, o(a) entrevistador(a) elegerá somente uma questão central (nomeada de questão norteadora), com a qual irá lançar a situação de entrevista. O desenvolvimento da entrevista dependerá fundamentalmente de como o(a) participante responderá a essa questão inicial. Isto é, o resultado da interação é definido em sua maior parte pelo(a) participante, em vez de ser guiado(a) por meio de um roteiro de temas e questões definidas a priori pelo(a) pesquisador(a) (Smith et al., 2009). Nesse sentido, essa modalidade de entrevista permite maior flexibilidade e plasticidade na interação entrevistado(a)-entrevistador(a), favorecendo um diálogo mais espontâneo e a exploração de assuntos mais particulares e complexos (Boni \& Quaresma, 2005).

Por sua vez, o(a) pesquisador(a) tem de estar atento(a) para a apreciação das prioridades e necessidades do(a) participante, ou seja, o senso de relatividade de importância dos assuntos que a pessoa entrevistada traz para o foco da entrevista. Igualmente, o(a) investigador(a) deve evitar ser diretivo(a) e conduzir o foco da entrevista, a não ser para a finalidade de fornecer o contexto para a exploração de temas gerais que, gradualmente, façam a transição para os específicos, a partir do que se traz o(a) participante efetivamente para a situação da entrevista (Smith et al., 2009).

Por essa razão, depois de disparada a questão norteadora, durante a continuidade da entrevista são formuladas questões de aprofundamento, cujo objetivo é possibilitar o esclarecimento (ou desvelamento) de facetas do assunto em foco, e buscar suscitar abertura para outras reflexões dos(as) participantes. Consequentemente, não se tratam de questões "prévias" à entrevista - e, portanto, exteriores à vivência da entrevista - mas inquietações que surgem no decorrer da interação entre o(a) pesquisador(a) e os(as) protagonistas.

Para ilustrar com um exemplo de aplicação, utilizamos em um de nossos estudos com configurações familiares homoparentais (Tombolato, 2019) uma entrevista aberta, que contemplou a seguinte questão central: "Contem-me como tem sido, para

5 Entende-se que a generalização estatística é aquela conferida por métodos estatísticos de análise, ao passo que a generalização teórica (ou indutiva) consiste na inferência de qualidades a partir da consideração de casos singulares, ou unidades de análise circunscritas, e sua generalização para outros casos ou unidades que, de fato, não foram objeto de observação e análise (Mattos, 2011). vocês, a experiência de conviver em família, desde o início até hoje" (para casais) e "Conte-me como é a experiência de conviver em família” (para as crianças). Sobre esse aspecto é válido refletir que, até há poucas décadas, a entrevista com a população infantil era uma técnica pouco utilizada e explorada pela literatura sobre metodologias de pesquisa:

(...) inclusive porque, usualmente, pensa-se a criança como incapaz de falar sobre suas próprias preferências, concepções ou avaliações. Com um conhecimento sobre a criança cada vez mais acurado, essa suposição tem sido questionada e tem sido explorado, crescentemente, o uso de entrevista com crianças (Carvalho, Beraldo, Pedrosa, \& Coelho, 2004, pp. 291-292).

Atualmente, encontramos um acervo significativo de pesquisas variadas que têm sido elaboradas a partir de dados oriundos de entrevistas com crianças e, particularmente, com entrevistas abertas utilizando-se a AFI (Back, Gustafsson, Larsson, \& Berterö, 2011; Paula, Padoin, Terra, Souza, \& Cabral, 2014).

Por fim, como é de praxe em outros enfoques de pesquisa qualitativa, as entrevistas são audiogravadas, mediante o consentimento prévio dos(as) participantes, e transcritas na íntegra e literalmente, respeitando-se o modo pessoal como cada participante se apropria e faz uso da linguagem como ferramenta de comunicação.

\section{Como operacionalizar e qual o senti- do de se fazer a análise de dados na perspectiva da AFI}

De acordo com os pressupostos balizadores da AFI, Smith et al. (2009) sugerem a inclusão de até seis participantes para que se assegure a realização de um bom estudo, enfatizando que três possa ser um número ideal. Evidentemente, esse número poderá variar de acordo com a complexidade e especificidades do fenômeno investigado. A definição da quantidade de participantes é apoiada no princípio geral de primar pela descrição idiográfica. Como um dos pressupostos é a redução dos(as) participantes a um contingente restrito, o(a) pesquisador(a) deve prestar atenção redobrada aos critérios de inclusão e exclusão na pesquisa, de modo a garantir que os indivíduos selecionados tenham as características desejáveis de um informante-chave. Isso significa que devem ser indivíduos (e possíveis participantes) com experiência no campo e no tema específico investigado.

O produto textual das transcrições das entrevistas de cada participante é analisado caso a caso por meio de análise sistemática e qualitativa. Esse material é então convertido em um relato narrativo, em que a interpretação analítica do(a) pesquisador (a) é apresentada em detalhes, embasada em trechos literais das entrevistas. Tendo em vista o número cir- 
cunscrito de participantes, tal como indicado pelos autores da AFI, é perfeitamente exequível gerenciar os dados de cada entrevista para eliciar, posteriormente, os temas preponderantes nas falas dos indivíduos do grupo.

Entretanto, se a quantidade for superior a seis participantes, resultando em um corpus amplo, inevitavelmente a análise de cada caso não poderá ser tão detalhada. Desse modo, a ênfase na análise nesse caso poderá voltar-se para a avaliação de quais foram os temas-chave emergentes para o grupo de participantes como um todo. Pode até acontecer o caso de que sejam identificados temas emergentes no nível da particularidade, mas que se postergue a busca por padrões e conexões até que se possam examinar todos os casos juntos. É possível que haja uma grande variedade em termos de detalhes particulares da análise e da ponderação relativa entre o nível grupal e o individual. Isso vai determinar até que ponto a análise caminha para uma particularidade e até que ponto caminha para uma análise de grupo. Todavia, mesmo quando a análise se concentrar principalmente no nível do grupo, o que a torna uma análise tipicamente AFI, os temas no nível grupal ainda podem ser ilustrados com exemplos particulares obtidos a partir das falas dos indivíduos (Smith et al., 2009).

Considerando ainda a perspectiva dos autores acerca de estudos com maior quantidade de participantes, a identificação da recorrência entre os casos é importante. A decisão principal, nesse momento, é como o status de tema recorrente é definido para um estudo em particular. Assim, por exemplo, pode-se considerar que, para que um tema superordenado emergente seja classificado como recorrente, ele deverá estar presente em pelo menos um terço, ou metade ou, mais rigorosamente falando, em todas as entrevistas dos(as) participantes. Esse tipo de "contagem” também pode ser considerada uma forma de incrementar a validade dos dados de um corpus extenso, mas não deve ser confundida com uma intenção subjacente de quantificar o fenômeno.

Segundo os autores, não há regra para definir que determinado achado identificado nos relatos dos(as) participantes é recorrente. A decisão é determinada por questões pragmáticas, tais como o produto final que se espera obter de um projeto de pesquisa. O grau de recorrência também será influenciado pelo nível que o(a) pesquisador(a) imprime à tematização e aos comentários sobre os dados obtidos. Um tema superordenado, expresso em um nível global, possivelmente terá mais ocorrências em um corpus de análise do que um tema expresso de maneira específica ou idiossincrática. Isso não quer dizer que um nível seja mais adequado do que o outro, tudo depende de como a análise evolui e do que se está tentado fazer com ela.

Além disso, também é importante considerar que a indicação de prevalência para um tema su- perordenado no grupo permite ainda uma variação considerável. Participantes diferentes podem manifestar o mesmo tema superordenador (mais abrangente) em diferentes temas. $\mathrm{O}$ tema superordenador pode parecer muito diferente no modo como ele é diferenciado pelos(as) participantes. Optar por aplicar a AFI a grande número de participantes frequentemente envolve a negociação quanto à relação entre convergência e divergência, entre o que é compartilhado e o que é individualizado (Smith et al., 2009).

Tendo identificado um conjunto de critérios que podem ser utilizados para identificar temas recorrentes, é recomendável como um passo final encontrar uma forma de mostrar graficamente as inter-relações entre os temas grupais recorrentes. Alguns dos processos descritos anteriormente, tais como abstração e subsunção (ancorar do tema mais geral para o mais específico) podem novamente ser uteis nessa situação. Estudos com maior quantidade de participantes requerem habilidade considerável do(a) pesquisador(a) para preservar um foco idiográfico na voz individual do(a) participante, ao mesmo tempo em que são feitas alegações (deduções) para o grupo maior.

Em suma, se a quantidade de participantes for administrável (aproximadamente seis), espera-se que trechos de falas sejam destacados e utilizados para ilustrar cada tema. Se o estudo tiver um número maior de participantes e/ou de temas sistematizados, provavelmente não será viável apresentar um trecho de cada participante para cada tema. Nesse caso, o(a) pesquisador(a) deverá selecionar com cautela alguns exemplos que considera apropriados para cada tema. Espera-se, dessa maneira, que cada tema seja embasado em situações comuns detectadas em um certo número de participantes e que, na narrativa geral, o(a) pesquisador(a) recorra aos relatos de um modo equilibrado, sem perder a perspectiva mais global da análise do fluxo verbal.

Nesse sentido, Carvalho et al. (2004), ao refletirem sobre a pesquisa qualitativa a partir das características da análise, concluem que:

(...) a análise qualitativa aparentemente contorna o problema da categorização intrínseco à análise quantitativa; no entanto, tanto quanto esta, requer recortes do fluxo verbal e atribuição de sentido às verbalizações, de forma a sistematizá-las de maneira compreensível e heurística; e, também da mesma forma que a categorização, requer explicitação de critérios para esses recortes e atribuições, para permitir o compartilhamento da análise e das conclusões com outros pesquisadores, uma condição necessária do processo de produção do conhecimento. Essa explicitação de critérios, no caso de análises qualitativas, exige muito mais do pesquisador, 
em termos de clareza conceitual, experiência e intimidade com os dados, do que uma definição compartilhável de categorias estanques (p. 297).

\section{Aplicações da AFI no cenário brasilei- ro: possibilidades e desafios}

A distribuição geográfica das pesquisas que utilizam a AFI segue um padrão similar de expansão. A maioria dos trabalhos iniciais se deu no Reino Unido, porém, pesquisadores(as) que utilizam a AFI podem ser encontrados(as) em qualquer parte do planeta. Não se surpreende que a maior parte desses estudos ocorra em países de língua inglesa, mas, obviamente, a AFI também é utilizada em lugares em que o inglês não é a primeira língua, por exemplo na Hungria (Kassai et al., 2017), Alemanha (Miebach et al., 2019) e Colômbia (Ramírez-Perdomo et al., 2018).

Nas publicações em periódicos brasileiros foram resgatados poucos estudos que utilizam a AFI como arcabouço teórico e/ou metodológico (Ferreira \& Lemos, 2016; Kimura, Maffioletti, Santos, Baptista, \& Dourado, 2015; Lima \& Miranda, 2018; Macedo, Marques, Queirós, \& Mariotti, 2018; Rabinovich \& Costa, 2010; Santos, Sousa, Ganem, Silva, \& Dourado, 2013; Stédile, Hartmann, \& Silva, 2014). As temáticas abordadas por essas pesquisas referem-se às áreas de saúde e religiosidade.

Um desses estudos mais recentes, realizado por Lima e Miranda (2018) na área da psicologia, apresentou uma reflexão sobre o trabalho de seis cuidadores de saúde inseridos em Residências Terapêuticas. A partir da transcrição e leituras das entrevistas, os autores identificaram temas que foram agrupados e reorganizados. Posteriormente, esses temas se tornaram uma categoria temática: "Vivências nas Residências", e com isso foram elaboradas narrativas que agruparam a interpretação e as falas dos participantes. Os autores descreveram brevemente a AFI e sua utilização em dois parágrafos da seção de Método de seu artigo.

Outro estudo, desenvolvido por Macedo et al. (2018) na área de terapia ocupacional, teve por objetivo conhecer e analisar as potencialidades e dificuldades de pessoas com transtorno esquizofrênico frente às Atividades Instrumentais da Vida Diária. Contou com 40 participantes, entre pessoas com diagnóstico de esquizofrenia, familiares e profissionais de saúde mental. Por meio dos dados transcritos a partir da realização de seis grupos focais, foram identificados três temas principais e nove subtemas. A referência à AFI apareceu em dois breves parágrafos do texto, na seção de método e nos resultados. Ainda que os autores tenham discutido os temas e subtemas, não evidenciaram como aplicaram os pressupostos da AFI, que propõe estudar em profundidade um número condensado de participantes, à coleta e análise dos grupos focais que totalizaram 40 pessoas.
Embora existam escassos estudos nacionais, constata-se que na comunidade científica internacional há numerosas pesquisas que se apoiam nesse referencial (AFI) já bem estabelecido no âmbito da metodologia qualitativa. Dentre essas pesquisas, conduzidas em diversas áreas, podemos destacar a psicologia do esporte (Lavallee \& Robinson, 2007), saúde (Marriott \& Thompson, 2008), sexualidade (Alexander \& Clare, 2004), bem como os estudos que investigam a própria teoria da AFI (Larkin et al., 2011).

Diante das perspectivas promissoras da aplicação da AFI, podemos pensar em possíveis cenários desafiadores para a prática teórico-investigativa. Um deles se refere à articulação entre os três campos teóricos e epistemológicos densos (fenomenologia, hermenêutica e idiografia), que possivelmente se constitui na tarefa mais árdua e ambiciosa do(a) investigador(a) que se orienta pela proposta da AFI. Outro exemplo de desafio que o(a) pesquisador(a) que faz uso da AFI deve estar pronto para lidar é como assegurar a credibilidade de seus achados. A confiabilidade seguramente é uma questão central na pesquisa qualitativa. Refere-se à credibilidade ou grau de confiança em determinada achado, o que em estudos quantitativos se denomina validade (Wu et al., 2016). O(a) pesquisador(a) em psicologia deve tomar uma série de cuidados metodológicos para assegurar à comunidade científica a confiabilidade de seus resultados.

Nesse aspecto, há diversas maneiras de se estabelecer a confiabilidade de um estudo, o que inclui métodos de naturezas variadas, tais como estratégias que permitam a um membro externo corroborar o ponto de vista do(a) pesquisador(a), seja por meio de interrogatório de pares, engajamento prolongado no campo, análise de casos negativos, auditabilidade, confirmação e bracketing - que é análogo à técnica utilizada em fotografia, que consiste em fotografar a mesma cena repetidas vezes, utilizando diferentes exposições (Guba \& Lincoln, 2005). Os métodos mais reconhecidos e comumente utilizados para incrementar a confiabilidade em pesquisas qualitativas são: a triangulação de dados e a obtenção de opinióes de diferentes entrevistados(as).

\section{Considerações Finais}

Este estudo apresentou os principais fundamentos e as potenciais aplicações da Análise Fenomenológica Interpretativa - AFI, um referencial teórico-metodológico recente, mas já bem estabelecido internacionalmente em pesquisas sociais de delineamento qualitativo, porém contando com um número ainda restrito de estudos publicados na realidade brasileira. Por meio de um recorte exploratório pretendeu-se contribuir não apenas para preencher uma lacuna que identificamos na literatura nacional, mas, principalmente, para ampliar o conhecimento dessa abordagem no contexto brasileiro, as- 
sim como inspirar e incentivar o desenvolvimento de novos estudos com essa abordagem qualitativa de pesquisa.

A pesquisa fenomenológica ocupa um lugar relevante entre as metodológicas qualitativas na psicologia brasileira. Diversos grupos de pesquisa nacionais têm se dedicado ao avanço do conhecimento nesse paradigma, o que justifica a disseminação de uma abordagem nova como a AFI para a ampla divulgação e aplicação em contextos diversificados, inspirando resultados promissores. Nesse sentido, aponta-se a necessidade de estudos futuros que contemplem determinados aspectos específicos da AFI no contexto brasileiro, como a tradução das obras de referência sobre a teoria para a língua portuguesa, os desafios e as discussões contemporâneas quanto aos limites e possibilidades de uso dessa abordagem, as áreas de pesquisa que poderiam se beneficiar com sua aplicação com base no que se encontrou nos estudos internacionais, assim como em novas alternativas para aplicação, discussão e crítica teórico-metodológica. Ressalta-se, a título de sugestão, a necessidade de mapear os principais desafios e interesses de pesquisa para que se possa ampliar o uso da AFI em pesquisas nacionais na área da psicologia.

\section{Referências}

Alexander, N., \& Clare, L. (2004). You still feel different: the experience and meaning of women's self injury in the context of a lesbian or bisexual identity. Journal of Community and Applied Social Psychology, 14(2), 70-84.

Amatuzzi, M. M. (1996). Apontamentos acerca da pesquisa fenomenológica. Estudos de Psicologia (Campinas), 13(1), 5-10.

Back, C., Gustafsson, P. A., Larsson, I., \& Berterö, C. (2011). Managing the legal proceedings: an interpretative phenomenological analysis of sexually abused children's experience with the legal process. Child Abuse \& Neglect, 35(1), 50-57.

Bogdan, R., \& Taylor, S. (1990). Looking at the bright side: a positive approach to qualitative policy and evaluation research. Qualitative Sociology, 13(2), 183-192.

Boni, V., \& Quaresma, S. J. (2005). Aprendendo a entrevistar: como fazer entrevistas em Ciências Sociais. Em Tese, 2(1), 68-80.

Carvalho, A. M. A., Beraldo, K. E. A., Pedrosa, M. I., \& Coelho, M. T. (2004). O uso de entrevistas em estudos com crianças. Psicologia em Estudo (Maringá), 9(2), 291-300.

Creswell, J. (2008). Research design: qualitative, quantitative, and mixed methods approaches. Thousand Oaks, CA: Sage.
Denzin, N. K., \& Lincoln, Y. S. (Eds.). (2005). The sage handbook of qualitative research ( $3^{\text {rd }}$ ed.). Thousand Oaks, CA: Sage.

Feijoo, A. M. L. C., \& Mattar, C. M. (2014). A fenomenologia como método de investigação nas filosofias da existência e na psicologia. Psicologia: Teoria e Pesquisa (Brasília), 30(4), 441-447.

Ferreira, R. M. B., \& Lemos, M. F. (2016). A mulher e o câncer de mama: um olhar sobre o corpo adoecido. Perspectivas em Psicologia (Uberlândia), (20)1, 178-201.

Fonseca, J. J. S. (2002). Metodologia da pesquisa científica. Fortaleza, CE: UEC.

Forghieri, Y. C. (1993). A investigação fenomenológica da vivência: justificativa, origem, desenvolvimento, pesquisas realizadas. Cadernos $d a$ ANPEPP, 19-42.

Forghieri, Y. C. (2004). Psicologia fenomenológica: Fundamentos, método e pesquisa (3a ed.). São Paulo: Pioneira.

Furlan, R. (2008). A questão do método na psicologia. Psicologia em Estudo (Maringá), 13(1), 25-33.

Gadamer, H. (1999). Verdade e método: traços fundamentais de uma hermenêutica filosófica (F. P. Meurer, Trad.). Rio de Janeiro, RJ: Vozes.

Giorgi, A., \& Sousa, D. (2010). Método fenomenológico de investigação em psicologia. Lisboa, Portugal: Fim de Século.

Guba, E. G., \& Lincoln, Y. S. (2005). Paradigmatic controversies, contradictions, and emerging influences. In N. K. Denzin \& Y. S. Lincoln (Eds.). The sage handbook of qualitative research ( $3^{\text {rd }} \mathrm{ed}$.), (pp. 191-215). Thousand Oaks, CA: Sage.

Heidegger, M. (2012). Ser e tempo (F. Castilho, Trad.). Rio de Janeiro, RJ: Vozes.

Husserl, E. (2012). A crise das ciências europeias e a fenomenologia transcendental: uma introdução à filosofia fenomenologia (D. F. Ferrer, Trad.). Rio de Janeiro, RJ: Forense Universitária.

Kassai, S., Pintér, J. N., Rácz, J., Erdősi, D., Milibák. R., \& Gyarmathy, V. A. (2017). Using Interpretative Phenomenological Analysis to assess identity formation among users of synthetic cannabinoids. International Journal of Mental Health and Addiction, 15(5), 1047-1054.

Kaurak, F. S., Manhães, F. C., \& Medeiros, C. H. (2010). Metodologia da pesquisa: um guia prático. Itabuna, BA: Via Litterarum.

Kimura, N. R. S., Maffioletti, V. L. R., Santos, R. L., 
Baptista, M. A. T., \& Dourado, M. C. N. (2015). Psychosocial impact of early onset dementia among caregivers. Trends in Psychiatry and Psychotherapy (Porto Alegre), 37(4), 213-219.

Larkin, M., Watts, S., Clifton, E. (2006). Giving voice and making sense in Interpretative Phenomenological Analysis. Qualitative Research in Psychology, 3(2), 102-120.

Larkin, M., Eatough, V., \& Osborn, M. (2011). Interpretative phenomenological analysis and embodied, active, situated cognition. Theory and Psychology, 21(3), 318-337.

Lavallee, D., \& Robinson, H. K. (2007). In pursuit of an identity: A qualitative exploration of retirement from women's artistic gymnastics. Psychology of Sport and Exercise, 8(1), 119-141.

Lima, L. A. B., \& Miranda, F. J. (2018). Análise do trabalho dos cuidadores em saúde nas Residências Terapêuticas. Psicologia \& Sociedade, 30, e174842.

Lindlof, T. R., \& Taylor, B. C. (2002). Qualitative communication research methods: second edition. Thousand Oaks, CA: Sage.

Lowenberg, J. S. (1993). Interpretative research methodology: broadening the dialogue. Advances in Nursing Science, 16(2), 57-69.

Lüdke, M., \& André, M. E. D. A. (1986). Pesquisa em educação: Abordagens qualitativas. São Paulo, SP: E. P.U.

Marriott, C., \& Thompson, A. R. (2008). Managing threats to femininity: personal and interpersonal experience of living with vulval pain. Psychology \& Health, 23(2), 243-258.

Macedo, M., Marques, A., Queirós, C., \& Mariotti, M. C. (2018). Esquizofrenia, atividades instrumentais de vida diária e funções executivas: uma abordagem qualitativa. Cadernos Brasileiros de Terapia Ocupacional, 26(2), 287-298.

Mattos, P. L. C. L. (2011). "Os resultados desta pesquisa (qualitativa) não podem ser generalizados": pondo os pingos nos is de tal ressalva. Cadernos EBAPE.BR, 9(spe 1), 450-468. doi:10.1590/ S1679-39512011000600002

Miebach, L., Wolfsgruber, S., Frommann, I., Fließbach, K., Jessen, F., Buckley, R. F., \& Wagner, M. (2019). Cognitive complaints in memory clinic patients and in depressive patients: an Interpretative Phenomenological Analysis. The Gerontologist, 59(2), 290-302.

Minayo, M. C. S. (Org.). (2010). Pesquisa social: teoria, método e criatividade. (29a ed.). Petrópolis, RJ: Vozes.

Minayo, M. C. S., \& Sanches, O. (1993). Quantitati- vo-qualitativo: oposição ou complementaridade? Cadernos de Saúde Pública, 9(3), 237-248.

Moreira, D. A. (2002). O método fenomenológico na pesquisa. São Paulo, SP: Thomsom Learning.

Packer, M. J., \& Addison, R. B. (1989). Entering the circle: hermeneutic investigation in psychology. New York, EUA: State University of New York Press.

Paula, C. C., Padoin, S. M. M., Terra, M. G., Souza, I. E. O., \& Cabral, I. E. (2014). Modos de condução da entrevista em pesquisa fenomenológica: relato de experiência. Revista Brasileira de Enfermagem, 67(3), 468-472.

Pernecky, T. (2016). Epistemology and metaphysics for qualitative research. London, UK: Sage.

Rabinovich, E. P., \& Costa, L. A. F. (2010). A “palavra divina" como logos separador. Psicologia em Estudo (Maringá), 15(2) 333-341.

Ramírez-Perdomo, C. A., Rodríguez-Velez, M. E., \& Perdomo-Romero, A. Y. (2018). Incertidumbre frente al diagnóstico de cáncer. Texto \& Contexto Enfermagem, 27(4), e5040017.

Ricoeur, P. (1970). Freud and Philosophy: an essay on interpretation. New Haven, EUA: Yale University Press.

Santos, R. L., Sousa, M. F. B., Ganem, A. C., Silva, T. V., \& Dourado, M. C. N. (2013). Cultural aspects in dementia: differences in the awareness of Brazilian caregivers. Trends in Psychiatry and Psychotherapy (Porto Alegre), 35(3), 191-197.

Savin-Baden, M., \& Major, C. (2013). Qualitative research: the essential guide to theory and practice. London, UK: Routledge.

Schmidt, L. K. (2012). Hermenêutica (F. Ribeiro, Trad.). Petrópolis, RJ: Vozes.

Smith, J. A. (1996). Beyond the divide between cognition and discourse: using interpretative phenomenological analysis in health psychology. Psychology \& Health, 11(2), 261-271.

Smith, J. A., Flowers, P., \& Larkin, M. (2009). Interpretative phenomenological analysis: theory, method and research. London, UK: Sage.

Stake, R. (1995). The art of case study research. Thousand Oaks, CA: Sage.

Stédile, A., Hartmann, F., \& Silva, L. D. (2014). O desenvolvimento do vínculo mãe bebê após o diagnóstico de Síndrome de Down. Saúde Mental em Foco do Cesuca, 2(1), 1-14.

Tombolato, M. A. (2019). As famílias homoparentais 
nas vozes de pais gays, mães lésbicas e seus/ suas filhos(as) (Tese de Doutorado). Programa de Pós-Graduação em Psicologia, Universidade de São Paulo, São Paulo.

Viesenteiner, J. L. (2013). O conceito de vivência ( $E r-$ lebnis) em Nietzsche: gênese, significado e recepção. Kriterion: Revista de Filosofia, 54(127), 141-155.

Wu, Y. P., Thompson, D., Aroian, K. J., McQuaid, E. L., \& Deatrick, J. A. (2016). Commentary: writing and evaluating qualitative research reports. Journal of Pediatric Psychology, 41(5), 493-450.

Mário Augusto Tombolato (orcid.org/0000-00022486-2671). Pós-Doutorando do Departamento de Psicologia Clínica, Instituto de Psicologia, da Universidade de São Paulo - São Paulo. Doutor pelo Programa de Pós-Graduação em Psicologia da Universidade de São Paulo, FFCLRP, USP, Ribeirão Preto, com estágio (sanduíche) no Centre for Family Research da Universidade de Cambridge, Reino Unido. Endereço institucional: Av. Professor Mello de Moraes, 1721 - sala 25, bloco F. Bairro: Cidade Universitária. Cidade: São Paulo, SP. CEP 05508-030. E-mail: marioaugt@hotmail.com

Manoel Antônio dos Santos (orcid.org/0000-00018214-7767). Professor Titular da Universidade de São Paulo (USP), Faculdade de Filosofia, Ciências e Letras de Ribeirão Preto e do Programa de Pós-Graduação em Psicologia. Membro do GT ANPEPP Psicologia e Relações de Gênero. Líder do grupo Laboratório de Ensino e Pesquisa em Psicologia da Saúde, registrado no Diretório de Grupos de Pesquisa do CNPq (CNPqUSP-FFCLRP-USP). É Editor Responsável da revista Paidéia (Qualis-CAPES A1) e Editor Associado da Revista da SPAGESP.

Este artigo é derivado da Tese de Doutorado desenvolvida pelo primeiro autor junto ao Programa de Pós-Graduação em Psicologia da Faculdade de Filosofia, Ciências e Letras de Ribeirão Preto, Universidade de São Paulo, sob orientação do segundo autor.

Apoio: Fundação de Amparo à Pesquisa do Estado de São Paulo (FAPESP), processos 2015/09173-0 e 2017/08547-0.

Recebido em 20.09.2018

Primeira Decisão Editorial em 07.01.2019

Segunda Decisão Editorial em 14.05.2019

Aceito em 26.08.2019 